\title{
RESISTIVE BALLOONING STABILITY OF ASDEX EQUILIBRIA
}

\section{H.P. Zehrfeld, K. Grassie}

\section{MAX-PLANCK-INSTITUT FUR PLASMAPHYSIK}

8046 GARCHING BEI MUNCHEN 


\title{
MAX-PLANCK-INSTITUT FŨR PLASMAPHYSIK \\ GARCHING BEI MŨNCHEN
}

\section{RESISTIVE BALLOONING STABILITY \\ OF ASDEX EQUILIBRIA}

\author{
H.P. Zehrfeld, K. Grassie
}

IPP $5 / 16$

August 1987

Die nachstehende Arbeit wurde im Rahmen des Vertrages zwischen dem Max-Planck-Institut für Plasmaphysik und der Europäischen Atomgemeinschaft über die Zusammenarbeit auf dem Gebiete der Plasmaphysik durchgeführt. 


\begin{abstract}
Experiments on the maximum attainable beta values of ASDEX discharges show that the limits for this parameter lie in the range of theoretical predictions. In a previous publication we have attempted to theoretically identify the corresponding instabilities. While no significant correlation to global plasma instabilities could be found, the two-dimensional MHD equilibria calculated on the basis of ASDEX experimental parameters turned out to be close to the marginal ideal ballooning limit. In the present work we extend this investigation to resistive ballooning modes. Separatrix bounded as well as limiter controlled plasma equilibria are considered. Because of the small aspect-ratio of ASDEX $(\mathrm{A} \simeq 4)$ all equilibrium as well as stability calculations are performed in full toroidal geometry. After the formulation of a system of four equations describing the resistive evolution of velocity and magnetic fields in the high-m stability limit in coordinate-invariant form and its Fourier approximation in the neighbourhood of a localization field line, we solve the resulting quasi-mode equations applying methods of finite-element discretization. We find complex growth rates $\gamma$ with positive real part for values of the toroidal mode number $n$ below 100 . Calculated values of $\operatorname{Re}\{\gamma\} \leq 10^{-3} / \tau_{A} \mathrm{~s}^{-1}$ (with $\tau_{A}$ being the Alfvén time) are small and therefore in agreement with the experimentally observed non-disruptive behaviour at the $\beta_{\mathrm{p}}$ - limit. Thus we believe that the characteristic signatures which govern ASDEX high- $\beta_{\mathrm{p}}$ discharges can be explained by resistive ballooning modes.
\end{abstract}




\section{Introduction}

Studies of ideal ballooning modes of MHD equilibria reconstructed from ASDEX experimental data have shown that in a plasma configuration with relatively small aspect-ratio $(A \approx 4)$ toroidal effects play an important role. The large differential displacement of the magnetic surfaces at high $\beta_{\mathrm{p}}$ and the effects of increased inverse rotational transform $\mathrm{q}$ and of shear in the neighbourhood of the separatrix require very accurate, genuinely toroidal reference equilibria and a correspondingly careful treatment of the stability equations. Detailed stability analyses under this aspect [1] prove ASDEX equilibria to be close to the beta- [2] as well as to the marginal ideal ballooning stability limit. However, the destabilizing potential of ideal ballooning modes turns out to be too small to account for the so-called "hard $\beta_{\mathrm{p}}$-saturation" observed at ASDEX. The approach to the $\beta_{\mathrm{p}}$-limit found there has been termed "hard saturation" because it is distinguished by the absence of both sudden disruptions and of gradual deterioration of confinement time over an extended range of beta. Rather, the confinement time deterioration sets in abruptly at beta-values only about $20 \%$ below the critical one.

In this paper we extend these investigations to resistive ballooning modes. We present, in coordinate-invariant form, a closed system of four equations describing the resistive evolution of velocity and magnetic fields in the high-m stability limit. Subsequently the Fourier approximation of this set, leading to the resistive ballooning equations of reference [3], is numerically solved. We formulate a variational approach to this boundary value problem in four dependent variables with real and imaginary parts of the growth rate as parameters. Stationary values of the corresponding Lagrangian $\mathrm{L}$ are associated with resistive modes. The resulting growth rates will be represented as functions of the dimensionless radial parameter $\mathrm{r} / \mathrm{a}$ defined by $\mathrm{r} / \mathrm{a}=\left(\mathrm{V} / \mathrm{V}_{\mathrm{B}}\right)^{1 / 2}$, the toroidal mode number $\mathrm{n}$ and $\mathrm{a}$ pressure gradient scaling factor $\mathrm{dp}_{\mathrm{M}} / \mathrm{dp}$. Here $\mathrm{V}$ is the volume enclosed by the magnetic surface under consideration, $V_{B}$ the total plasma volume, $p$ the equilibrium pressure and $p_{M}$ the marginally-scaled pressure. $p_{M}$ is introduced for the study of pressure effects without calculating new MHD equilibria. For given pressure gradient scale factor $d p_{M} / d p$ the actual values which we obtained for these functions are uniquely determined by the following computational input: 
- Radial profiles for pressure $p$ and toroidal current I as functions of the volume V. They are calculated using experimental data in TRANSP code interpretation;

- Resistivity distributions with respect to the variable $\mathrm{V}$ which we calculate using radial profiles for inverse rotational transform $q$, electron density $n_{e}$, electron temperature $\mathrm{T}_{\mathrm{e}}$ and $\mathrm{Z}_{\text {eff }}[4]$.

All equilibrium as well as stability calculations are performed without any restriction on the aspect-ratio. We use MKSA units throughout.

\section{Theory}

For sufficiently large values of the poloidal mode number $m$ the resistive MHD equations predict the following linear evolution of the velocity field $\tilde{\mathbf{v}}$ and the magnetic induction $\tilde{\mathbf{B}}$ from their values in the equilibrium state:

$$
\begin{aligned}
& \tilde{\mathbf{v}}=\frac{1}{\mathbf{B}^{2}}(\tilde{\mathbf{v}} \cdot \mathbf{B B}+\mathbf{B} \times(\mathbf{B} \times(\nabla \tilde{\Phi} \times \nabla \sigma))) \\
& \tilde{\mathbf{B}}=\frac{\mu_{0}}{\mathbf{B}^{2}}(-\tilde{\mathbf{p}} \mathbf{B}+\mathbf{B} \times(\mathbf{B} \times(\nabla \tilde{\mathbf{A}} \times \nabla \sigma))) .
\end{aligned}
$$

Here $\sigma$ is the coordinate along the equilibrium magnetic field $\mathbf{B}$ with $\mathrm{d} \sigma=\mathrm{ds} / \mathrm{B}, \mathrm{s}$ being the field line arc length. $\tilde{\mathbf{v}} \mathbf{B} / \mathrm{B}$ is the parallel velocity, $\tilde{\Phi}$ the electric potential of the perturbation, $\tilde{A}$ a vector-potential like quantity of dimension $\mathrm{AVs} / \mathrm{m}^{2}$ and $\tilde{\mathbf{p}}$ the pressure perturbation. The evolution of these four field scalars is determined by the solution of the following closed set of equations:

$$
\begin{gathered}
\frac{1+\beta}{\left(\mathrm{c}_{\mathrm{p}} / \mathrm{c}_{\mathrm{v}}\right) \mathrm{p}} \frac{\partial \tilde{\mathrm{p}}}{\partial \mathrm{t}}=\left(2 \mathrm{k}-\frac{1+\beta}{\left(\mathrm{c}_{\mathrm{p}} / \mathrm{c}_{\mathrm{v}}\right) \mathrm{p}} \nabla \mathrm{p}\right) \cdot(\nabla \sigma \times \nabla \tilde{\Phi})-\mathbf{B} \cdot \nabla\left(\frac{\tilde{\mathrm{v}} \cdot \mathrm{B}}{\mathrm{B}^{2}}\right)+\frac{1}{\mathrm{~B}^{2}} \eta \Delta \tilde{\mathrm{p}} \\
\rho \frac{\partial(\tilde{\mathrm{v}} \cdot \mathrm{B})}{\partial \mathrm{t}}=-\mu_{0}(\nabla \sigma \times \nabla \tilde{\mathrm{A}}) \cdot \nabla \mathrm{p}-\mathbf{B} \cdot \nabla \tilde{\mathrm{p}} \\
\mu_{0} \frac{\partial \tilde{\mathrm{A}}}{\partial \mathrm{t}}=\mathbf{B} \cdot \nabla \tilde{\Phi}+\eta \Delta \tilde{\mathrm{A}} \\
\frac{\rho}{\mathrm{B}^{2}} \Delta\left(\frac{\partial \tilde{\Phi}}{\partial \mathrm{t}}\right)=\mathbf{B} \cdot \nabla\left(\frac{1}{\mathrm{~B}^{2}} \Delta \tilde{\mathrm{A}}\right)+2 \mathbf{k} \cdot(\nabla \tilde{\mathrm{p}} \times \nabla \sigma) .
\end{gathered}
$$


$\beta$ is a local beta value and $\mathbf{k}$ the curvature vector:

$$
\beta=\frac{\mu_{0}\left(\mathrm{c}_{\mathrm{p}} / \mathrm{c}_{\mathrm{v}}\right) \mathrm{p}}{\mathrm{B}^{2}}, \quad \mathbf{k}=(\mathbf{B} \cdot \nabla(\mathrm{B} / \mathrm{B})) / \mathrm{B}
$$

$\Delta$ is the Laplacian, $\mathrm{c}_{\mathrm{p}} / \mathrm{c}_{\mathrm{v}}$ the ratio of the specific heats, $\eta$ the resistivity and $\rho$ the mass density. Assuming a time-dependence $\sim \exp \{\gamma \mathrm{t}\}$ of the perturbed quantities with complex growth rate $\gamma$, and Fourier representing them in the neighbourhood of a localization field line leads to the stability criterion formulated in [3]:

The plasma is unstable with respect to resistive ballooning modes if there are square-integrable solutions $\mathrm{u}$ and $\mathrm{v}$ of

$$
\begin{gathered}
\mathbf{B} \cdot \nabla\left\{\frac{\left(1+\mathrm{S}^{2}\right)}{\mathrm{D}|\nabla \Psi|^{2}} \mathbf{B} \cdot \nabla \mathrm{u}\right\}+\left(2 \mu_{0} \frac{\mathrm{dp}}{\mathrm{d} \Psi} \frac{\mathrm{k}_{\mathrm{g}} \mathrm{S}-\mathrm{k}_{\mathrm{n}}}{|\nabla \Psi|}-\mu_{0} \rho \gamma^{2} \frac{1+\mathrm{S}^{2}}{|\nabla \Psi|^{2}}\right) \mathrm{u}+2 \mu_{0} \frac{\mathrm{dp}}{\mathrm{d} \Psi} \frac{\mathrm{k}_{\mathrm{g}} \mathrm{S}-\mathrm{k}_{\mathrm{n}}}{|\nabla \Psi|} \mathrm{v}=0 \\
\mathbf{B} \cdot \nabla\left\{\frac{1}{\mathrm{~B}^{2}} \mathrm{~B} \cdot \nabla \mathrm{v}\right\}-2 \mu_{0} \frac{\mathrm{dp}}{\mathrm{d} \Psi}\left(\frac{\mathrm{n}^{2} \eta}{\mu_{0} \gamma}+\frac{\rho \gamma^{2}}{\mu_{0}(\mathrm{dp} / \mathrm{d} \Psi)^{2}}\right) \frac{\mathrm{k}_{\mathrm{g}} \mathrm{S}-\mathrm{k}_{\mathrm{n}}}{|\nabla \Psi|} \mathrm{u} \\
-\left\{\frac{\mathrm{n}^{2} \eta}{\mu_{0} \gamma}\left(2 \mu_{0} \frac{\mathrm{dp}}{\mathrm{d} \Psi} \frac{\mathrm{k}_{\mathrm{g}} \mathrm{S}-\mathrm{k}_{\mathrm{n}}}{|\nabla \Psi|}+\mu_{0} \rho \gamma^{2} \frac{1+\mathrm{S}^{2}}{|\nabla \Psi|^{2}}\right)+\frac{\mu_{0} \rho \gamma^{2}}{\mathrm{~B}^{2}} \frac{1+\beta}{\beta}\right\} \mathrm{v}=0
\end{gathered}
$$

on that line with Real $\{\gamma\}>0$. Here $\mathrm{k}_{\mathrm{g}}$ and $\mathrm{k}_{\mathrm{n}}$ are the projections of $\mathbf{k}$ in geodesic and normal directions, respectively, and

$$
\mathrm{D} \equiv 1+\frac{\mathrm{n}^{2} \eta}{\mu_{0} \gamma} \frac{\mathrm{B}^{2}\left(1+\mathrm{S}^{2}\right)}{|\nabla \Psi|^{2}}
$$

$\Psi$ is the poloidal flux of the magnetic field vanishing at $R=0$ in $(R, \varphi, z)-$ coordinates with $\mathrm{R}$ being the radial distance from - and $\varphi$ the angle around the axis of symmetry in $\mathrm{z}$-direction. $\mathrm{S}$ is the local shear, a secular quantity given by

$$
\mathrm{S}\left(\Psi, \Theta, \Theta_{0}\right) \equiv \frac{|\nabla \Psi|^{2}}{\mathrm{~B}} \int_{\Theta_{0}}^{\Theta} \frac{1}{|\nabla \Psi|^{4}}(\mathbf{B} \times \nabla \Psi) \cdot \operatorname{rot}(\mathrm{B} \times \nabla \Psi) \frac{\mathrm{d} \Theta^{\prime}}{\mathbf{B} \cdot \nabla \Theta^{\prime}}
$$

where $\Theta$ is any angle-coordinate along B. $\Theta_{0}$ is an arbitrary start value representing a free parameter in the stability investigation.

Prior to the solution of the stability equations (8-9) the equilibrium problem

$$
\mathrm{R}^{2} \operatorname{div} \frac{\nabla \Psi}{\mathrm{R}^{2}}+\mu_{0}^{2} \mathrm{JJ}^{\prime}(\Psi)+4 \pi^{2} \mu_{0} \mathrm{R}^{2} \mathrm{p}^{\prime}(\Psi)=0
$$




$$
\mathrm{JJ}^{\prime}(\Psi)=\frac{4 \pi^{2}}{\mu_{0}}\left\langle 1 / \mathrm{R}^{2}\right\rangle^{-1}\left(\frac{\mathrm{dI}}{\mathrm{dV}}-\mathrm{p}^{\prime}(\Psi)\right)
$$

for given profiles $p$ and $I$ as functions of $r / a=\left(V / V_{B}\right)^{1 / 2}$ must be solved for $\Psi$ and $\mathrm{J}$, where $\mathrm{J}$ is the poloidal current (the equivalent of $\Psi$ with respect to the current density field $\mathbf{j}) .\langle\ldots\rangle$ denotes the usual flux surface average so that

$$
\left\langle 1 / \mathrm{R}^{2}\right\rangle=\frac{\mathrm{d}}{\mathrm{dV}} \int_{\mathrm{V}} \frac{\mathrm{d}^{3} \mathrm{x}}{\mathrm{R}^{2}} .
$$

Introducing an external conductor system with $M$ currents $\left\{I_{k}\right\}_{k=1, M}$ at positions $\left\{x_{k}=R_{k} \nabla R+z_{k} \nabla z\right\}_{k=1, M}$ the free-boundary solution of (12-13) consistent with this system can be brought into the form

$$
\begin{gathered}
\Psi(\mathbf{x})=\Psi^{*}(\mathbf{x})+\frac{1}{4 \pi^{2}} \iint_{\partial \mathcal{R}} \frac{\mathrm{G}\left(\mathbf{x}, \mathbf{x}^{\prime}\right)}{\mathrm{R}^{\prime 2}} \nabla^{\prime} \Psi^{*}\left(\mathbf{x}^{\prime}\right) \cdot \mathrm{d} \mathbf{S}^{\prime}+\mu_{0} \sum_{\mathbf{k}=1}^{\mathrm{M}} \mathrm{G}\left(\mathbf{x}, \mathbf{x}_{\mathbf{k}}\right) \mathrm{I}_{\mathbf{k}} \\
\mathrm{G}\left(\mathbf{x}, \mathbf{x}^{\prime}\right) \equiv \frac{1}{4 \pi^{2}} \oint_{C} \oint_{C^{\prime}} \frac{\mathrm{d} \mathbf{x} \cdot \mathrm{d} \mathbf{x}^{\prime}}{\left|\mathbf{x}-\mathbf{x}^{\prime}\right|},
\end{gathered}
$$

where $\Psi^{*}$ is the solution of (12-13) satisfying the boundary condition $\Psi^{*} \mid \partial R=0$ with $R$ being a region with rectangular poloidal cross-section enclosing the plasma. The integrals entering the expression for Green's function $\mathrm{G}$ of the vacuum solution require integrations along toroidal circular lines $C$ and $C^{\prime}$ through $x=R \nabla R+z \nabla z$ and $\mathbf{x}^{\prime}=\mathrm{R}^{\prime} \nabla \mathrm{R}+\mathbf{z}^{\prime} \nabla \mathbf{z}$, respectively.

The magnetic field $\mathbf{B}$ then follows from the representation

$$
\mathbf{B}=\frac{1}{2 \pi}\left(\nabla \Psi \times \nabla \varphi+\mu_{0} \mathrm{~J} \nabla \varphi\right)
$$

thus, except for the growth rate $\gamma$, all quantities entering the coefficients of the stability equations (8-9) are determined. Furthermore the poloidal Alfvén transit time

$$
\tau_{\mathrm{A}} \equiv \frac{\left(2 \pi \mathrm{R}_{\mathrm{A}}\right)^{2} \rho}{\mu_{0} \mathrm{~J}^{2}}
$$

is given, where $\rho$ and $\mathrm{J}$ are to be taken on the considered magnetic surface; $\mathrm{R}_{\mathrm{A}}$ denotes the radial distance of the magnetic axis from the axis of symmetry. In what follows we refer to a normalized growth rate $\gamma$ which is obtained by multiplication with $\tau_{\mathbf{A}}$. 
The equations (8-9) can be seen to be equivalent to the stationarity conditions of the quadratic functional

$$
\mathrm{L}\left(\gamma, \Psi, \Theta_{0}\right)=\int_{-\infty}^{+\infty} \mathcal{L}\left(\gamma, \Psi, \Theta, \Theta_{0}, \mathbf{u}(\Theta), \dot{\mathbf{u}}(\Theta)\right) \mathrm{d} \Theta
$$

with respect to $\mathbf{u}$, with the Lagrange density

$$
\mathcal{L}=\frac{1}{2}\left(\dot{\mathbf{u}}^{\mathrm{T}} \cdot \mathbf{P} \cdot \dot{\mathbf{u}}-\mathbf{u}^{\mathrm{T}} \cdot \mathbf{Q} \cdot \mathbf{u}\right)
$$

$u=\left(u^{1}, u^{2}, u^{3}, u^{4}\right)$ comprises real and imaginary parts of $u$ and $v$ in equations (8-9) (so that $\mathrm{u}^{*}=\left(\mathrm{u}^{1}, \mathrm{u}^{2}\right)$ and $\mathrm{v}^{*}=\left(\mathrm{u}^{3}, \mathrm{u}^{4}\right)$ ), and $\dot{\mathrm{u}}=\mathrm{du} / \mathrm{d} \Theta$ the components $\mathrm{du}^{\mathrm{k}} / \mathrm{d} \Theta, \mathrm{k}=1, \ldots, 4$. $\mathbf{P}$ and $\mathbf{Q}$ are equilibrium determined real symmetric matrices with nonlinear dependence on the the complex growth rate $\gamma$. They have the structure

$$
\mathbf{P}=\left(\begin{array}{cccc}
\mathrm{P}_{11} & \mathrm{P}_{12} & 0 & 0 \\
\mathrm{P}_{12} & -\mathrm{P}_{11} & 0 & 0 \\
0 & 0 & \mathrm{P}_{33} & \mathrm{P}_{34} \\
0 & 0 & \mathrm{P}_{34} & -\mathrm{P}_{33}
\end{array}\right), \mathbf{Q}=\left(\begin{array}{cccc}
\mathrm{Q}_{11} & \mathrm{Q}_{12} & \mathrm{Q}_{13} & 0 \\
\mathrm{Q}_{12} & -\mathrm{Q}_{11} & 0 & -\mathrm{Q}_{13} \\
\mathrm{Q}_{13} & 0 & \mathrm{Q}_{33} & \mathrm{Q}_{34} \\
0 & -\mathrm{Q}_{13} & \mathrm{Q}_{34} & -\mathrm{Q}_{33}
\end{array}\right)
$$

and the matrix elements can be obtained by comparing the four Euler-Lagrange equations of (19) with real and imaginary parts of equations (8-9). Explicit expressions for these dimensionless quantities are given in the Appendix. The homogeneous boundary conditions on $\mathbf{u}$ of the corresponding two-point boundary value problem read

$$
\lim _{\Theta \rightarrow \pm \infty} \mathbf{u}(\Theta)=0
$$

Thus unstable resistive ballooning modes $u$ satisfying (22) are those stationary points of L with Real $\{\gamma\}>0$. 


\section{Computational Procedure}

On a given magnetic surface $\Psi=\Psi(\mathrm{R}, \mathrm{z})$ we define along the localization field line $\Theta:=\angle\left(\nabla R,\left(R-R_{A}\right) \nabla R+z \nabla z\right)$ and, according to the up-down symmetry of the ASDEX plasma configuration, $\Theta_{0}=0$. Let us consider the Lagrangian (19) on the finite interval $\left[-\Theta_{M},+\Theta_{M}\right]$ with sufficiently large $\Theta_{M}>0$ and $N+1$ grid points on $\left[-\Theta_{M},+\Theta_{M}\right]$. Then, representing $u$ by the $4(N+1)$ function values $\left\{\mathrm{x}^{\mu}\right\}_{\mu=1,4(\mathrm{~N}+1)}:=\left\{\left\{\mathrm{u}^{\mathrm{k}}\left(\Theta_{\mathrm{j}}\right)\right\}_{\mathrm{k}=1,4}\right\}_{\mathrm{j}=1, \mathrm{~N}+1}$, finite element discretization of (19) leads to $4(\mathrm{~N}+1)$ homogeneous equations for $\mathrm{x}$

$$
\mathbf{S} \cdot \mathbf{x}=\mathbf{0}, \quad \mathbf{S}=\mathbf{S}(\gamma)
$$

with the real symmetric system matrix $\mathbf{S}$, depending non-linearly on the complex growth rate $\gamma$. $S$ is block tridiagonal, the 4-by-4 blocks consisting of linear combinations of the matrices $\mathbf{P}$ and $\mathbf{Q}(21)$, evaluated at certain $\Theta$-values according to the chosen finite element discretization. The boundary conditions (22) are taken into account by dropping the first and last four columns and rows of $\mathbf{S}$, respectively.

In order to find those values of $\boldsymbol{\gamma}$ for which $\mathbf{S} \cdot \mathbf{x}=\mathbf{0}$ has non-trivial solutions $\mathbf{x}$, we have applied two methods:

The first one, which we preferentially used for ideal ballooning calculations, is based on $\mathrm{LDL}^{\mathrm{T}}$-decompositions of $\mathrm{S}[5,6]$, i.e. nonsingular congruence transformations of $\mathbf{S}(\gamma)$ into diagonal matrices $\mathbf{D}(\gamma)$, which preserve the number of positive and negative eigenvalues $\lambda$. If at least one element in $\mathbf{D}(\gamma)$ changes sign at $\gamma=\gamma_{0}$, then the eigenvalue of $\mathbf{S}$ of smallest absolute value has passed 0 , so that $\operatorname{det}\left\{\mathbf{D}\left(\gamma_{0}\right)\right\}=\operatorname{det}\left\{\mathbf{S}\left(\gamma_{0}\right)\right\}=0$. One has to keep in mind, however, that the entries in $\mathbf{D}$ correspond to four real eigenfunctions. Therefore $\mathbf{D}$ has to be analyzed in an ordered structure according to the four equations and eigenfunctions under consideration.

The second method uses inverse vector iteration $[7,8]$. In resistive calculations, especially in overstable situations, it turns out that this iterative determination of the eigenvalue of smallest absolute value is more efficient. However, since $\min \{|\lambda|: \lambda$ eigenvalue $\}$ is not a smooth function of $\gamma$, it is somewhat difficult to automatize the search for a solution of the system (23) - in other words : initial $\gamma$-values close to the final solution are necessary for an iterative search. 
For both methods sparse matrix techniques $[6,9]$ as well as buffered I/O operations have been applied in order to optimize the memory and computational time requirements with respect to the parameters $N$ and $\Theta_{M}$.

For an accumulated mesh in regions, where eigenfunctions vary strongly, i.e. at $\left\{\Theta_{\mathrm{k}}= \pm \mathrm{k} \pi \forall \mathrm{k} \leq \Theta_{\mathrm{M}} / \pi\right\}$, we typically need about $\mathrm{N}=300-500$ grid-points for $\Theta_{M}=150$. In some situations, e.g. marginal cases with small growth rates $\gamma<$ $10^{-3}$ or small resistivity, the necessary $\Theta_{M}$-value can be as large as 500 .

\section{Numerical Results}

All results which will be discussed in the following are for the high-beta discharge \#17005 at the time when $\beta_{\mathrm{p}}$ has reached its maximum value. Corresponding TRANSP-code determined pressure (p) and toroidal current (I) profiles are shown in Fig.1.
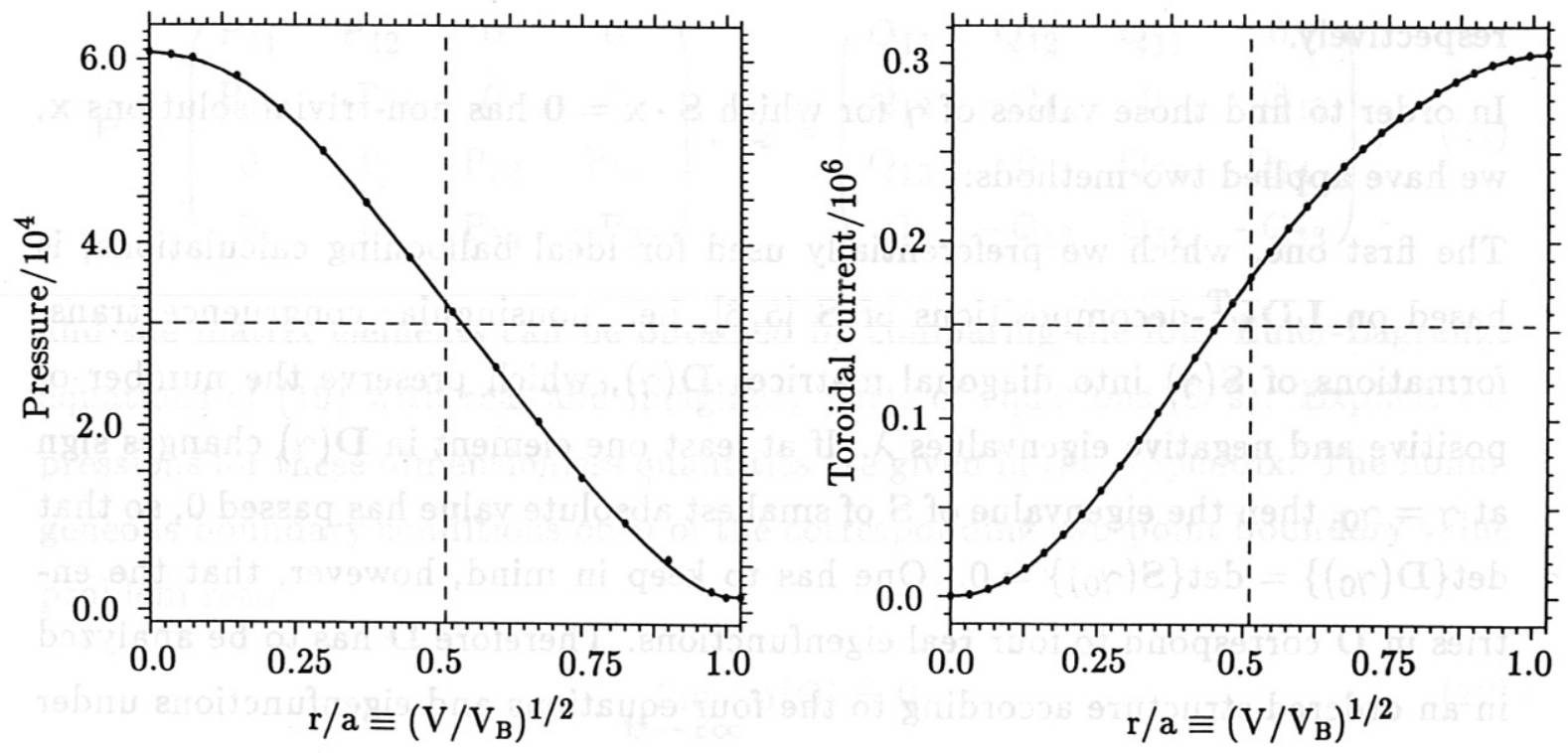

Fig. 1: Pressure and toroidal current profiles for the equilibrium of \#17005 at the time when $\beta_{\mathrm{p}}$ has reached its maximum.

These profiles serve as input for a subsequent two-dimensional equilibrium calculation and determine - together with the ASDEX conductor system - the magnetic flux $\Psi(R, z)$ according to equation (15).

Fig. 2 shows equilibrium contour lines for separatrix-defined and limiter-controlled 

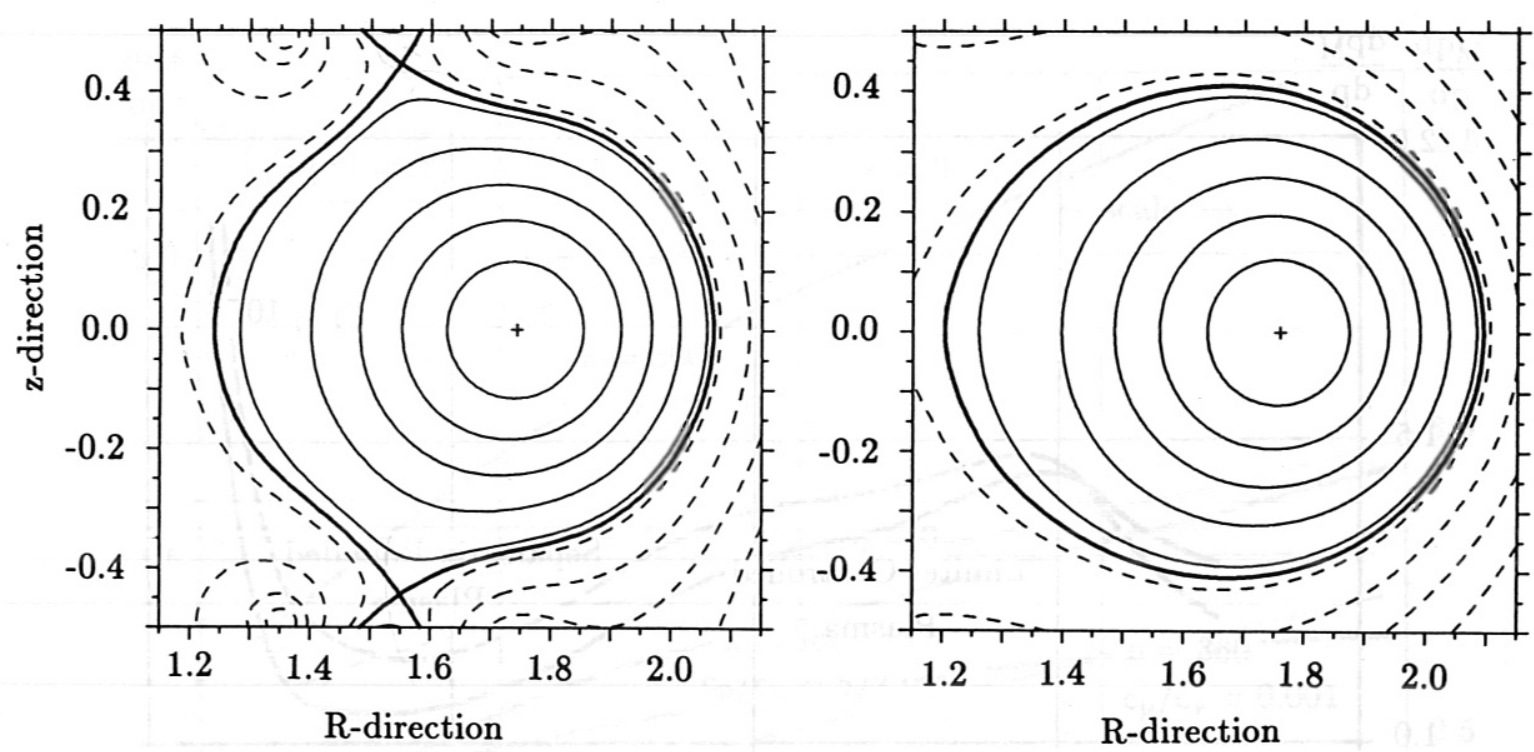

Fig. 2: ASDEX equilibrium flux surfaces corresponding to the profiles of Fig.1 for a separatrix bounded plasma (left) and a limiter controlled configuration (right).

plasma configurations, respectively. To produce these results and all data required for the stability investigations, we have used a special version of the Garching flow equilibrium code NIVA for the calculation of static, toroidal-current profile determined plasma equilibria.

Ideal ballooning stability results are shown in Fig.3, where the pressure multiplication factor $\mathrm{dp}_{\mathrm{M}} / \mathrm{dp}$ is plotted as a function of $\mathrm{r} / \mathrm{a}=\left(\mathrm{V} / \mathrm{V}_{\mathrm{B}}\right)^{1 / 2}$ at the maximum attained value of $\beta_{\mathrm{p}}$. It can be seen that the discharge is ideally ballooning stable on all flux surfaces, i.e. $\mathrm{dp}_{\mathrm{M}} / \mathrm{dp}$ exceeds 1 throughout, with a minimal value of $\simeq 1.15$ in the case of a separatrix-defined equilibrium (short dashed curve). A comparison with results for the limiter-controlled equilibrium (solid line) proves the latter to be more ballooning unstable, especially at larger radii where the stabilizing influence of shear is larger in the separatrix-bounded case.

The destabilizing influence of resistivity is demonstrated in Fig.4 for the particular magnetic surface at $r / a=\left(V / V_{B}\right)^{1 / 2} \simeq 0.67$ with $q \simeq 2$, where $d p_{M} / d p$ is plotted versus the purely real growth rate $\gamma$. Short dashed curves represent ideal ballooning results for the first stable regime (left scale) and the second stable regime (right scale), respectively, and have to be compared with resistive calculations (solid lines). The $\eta$-value of $3.32 \times 10^{-8} \mathrm{Vm} / \mathrm{A}$ for this surface and the 


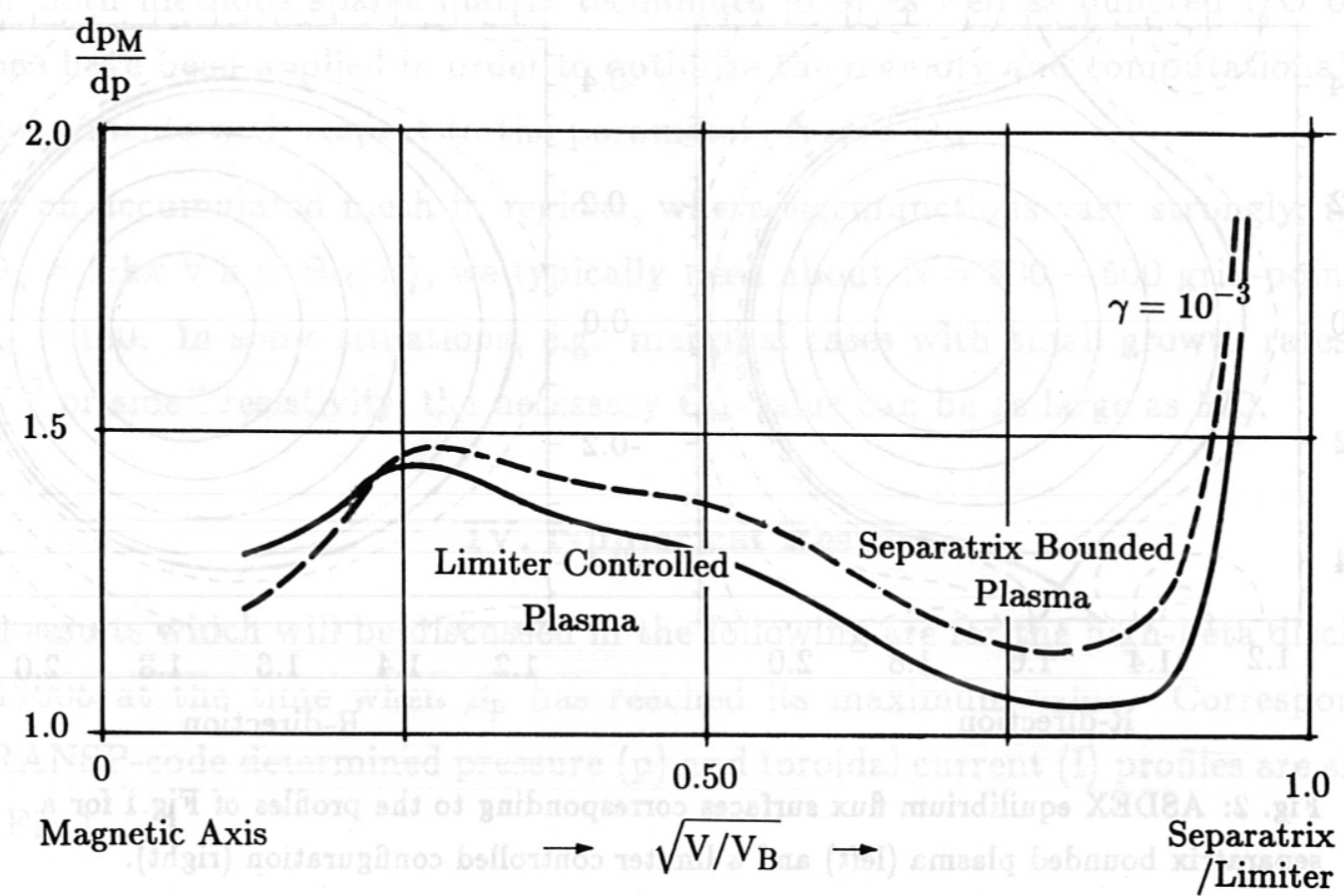

Fig. 3: Pressure gradient multiplication factor $d_{p_{M}} / d p$ versus normalized radius $r / a=\left(V / V_{B}\right)^{1 / 2}$ due to ideal ballooning modes. The dashed line represents results for the separatrix defined equilibrium of \#17005. For comparison calculations for the corresponding limiter controlled equilibrium are given by the solid curve.

relatively high ideal ballooning stability of ASDEX (see Fig.3) require an anomalously large toroidal mode number $\mathrm{n}>300$ to make resistivity effects significantly reduce $d p_{M} / d p$. It is interesting to note that for small growth rates a minimum in $\mathrm{dp}_{\mathrm{M}} / \mathrm{dp}$ versus $\operatorname{Re}\{\gamma\}$ is observed. Due to the stabilizing influence of compressibility, which increases with increasing resistivity, this minimum in $\mathrm{dp}_{\mathrm{M}} / \mathrm{dp}$ moves to even smaller values of $\gamma$, when $\mathrm{n}$ is decreased. The stabilizing influence of compressibility is separately demonstrated by the dot-dashed curve in Fig.5, representing a case with vanishing ratio of the specific heats, $c_{p} / c_{v} \rightarrow 0$. In this region of parameter space, i.e. at small growth rates or small resistivity, we find the corresponding eigenfunctions to be largely extended in Fourier space. As has been pointed out in [10], the increasing extension in Fourier space with decreasing importance of destabilizing terms can be explained by the larger region which is necessary for the mode to overcome the influence of the stabilizing terms.

The scaling of the growth rate with toroidal mode number $\mathrm{n}$ is presented in Fig.5 for two different values $d p_{M} / d p=1.5$ (upper curve) and $d p_{M} / d p=1$ (lower curve), 


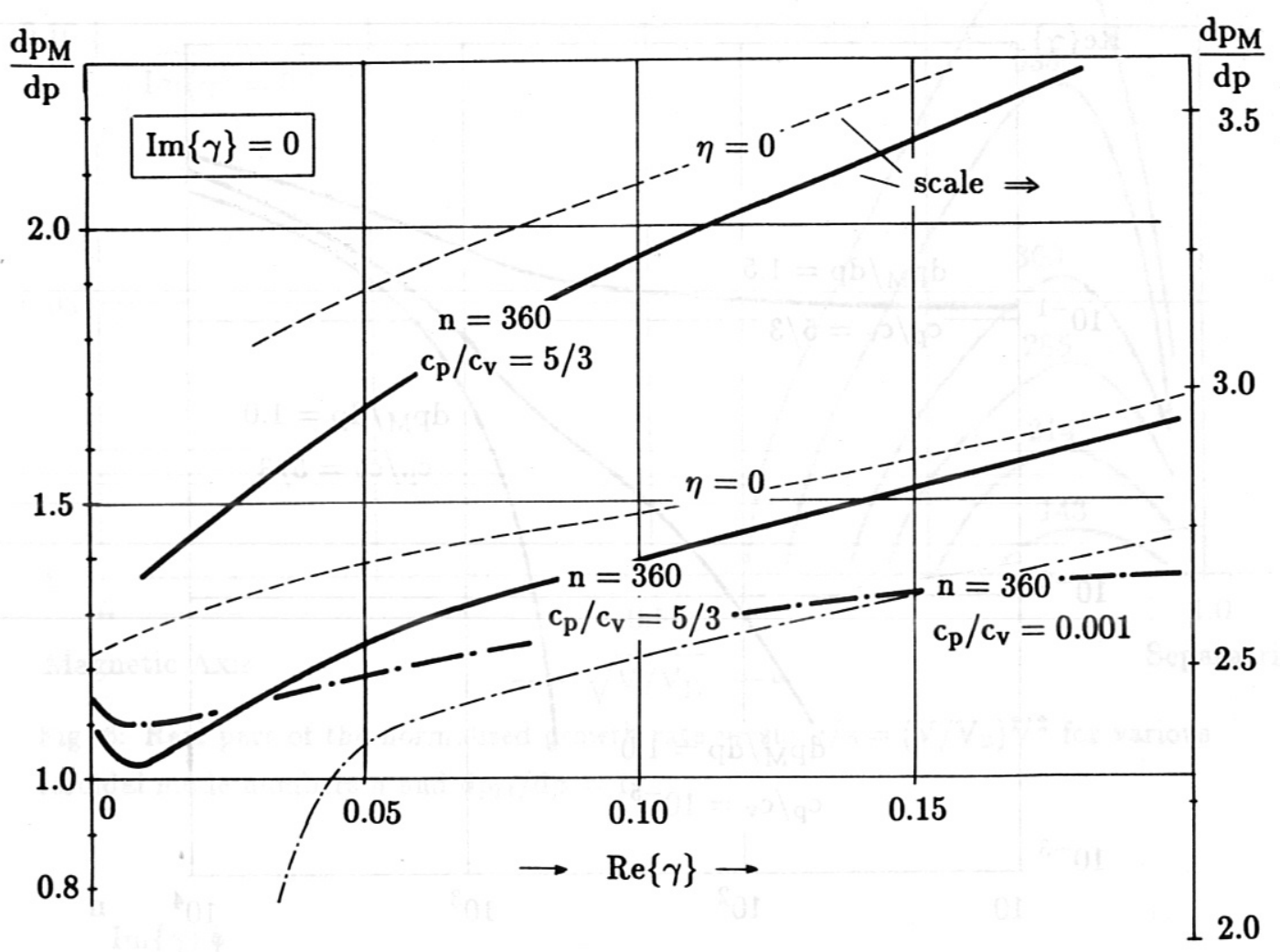

Fig. 4: $d p_{M} / d p$ versus real part of the normalized growth rate on the 14th flux surface (out of 32) at $r / a \simeq 0.67(q \simeq 2)$ for resistive ballooning modes. The neoclassical parallel resistivity on this flux surface is $3.32 \times 10^{-8} \Omega \mathrm{m}$. The solid lines represent resistive ballooning results with a toroidal mode number $n=360$ for the first (left scale) and the second (right scale) stability regime, respectively. For comparison ideal results are given by thin short-dashed curves. The thin dot-dashed line corresponds to a resistive calculation with vanishing compressibility. A result for smaller mass density ( $\rho$ is reduced by $90 \%$ ) is given by the dot-dashed curve.

respectively. Since a case which is ideally stable for $d_{M} / d p=1$ requires a certain threshold in $\mathrm{n}$ for positive growth rates to exist, the scaling at small toroidal mode numbers strongly depends on the chosen $\mathrm{dp}_{\mathrm{M}} / \mathrm{dp}$ value. Even for large toroidal mode numbers ( $\mathrm{n}>500$ ), where both curves approach each other, no simple power scaling law can be found. The central curve curve in Fig. 5 again corresponds to the limit of vanishing compressibility, where the growth rate scales like $\eta^{1 / 2}$ in the high temperature region and differences between compressible and incompressible results decrease with increasing $\gamma$ (or increasing $\mathrm{n}$ ), i.e. when modes are more localized in Fourier space. 


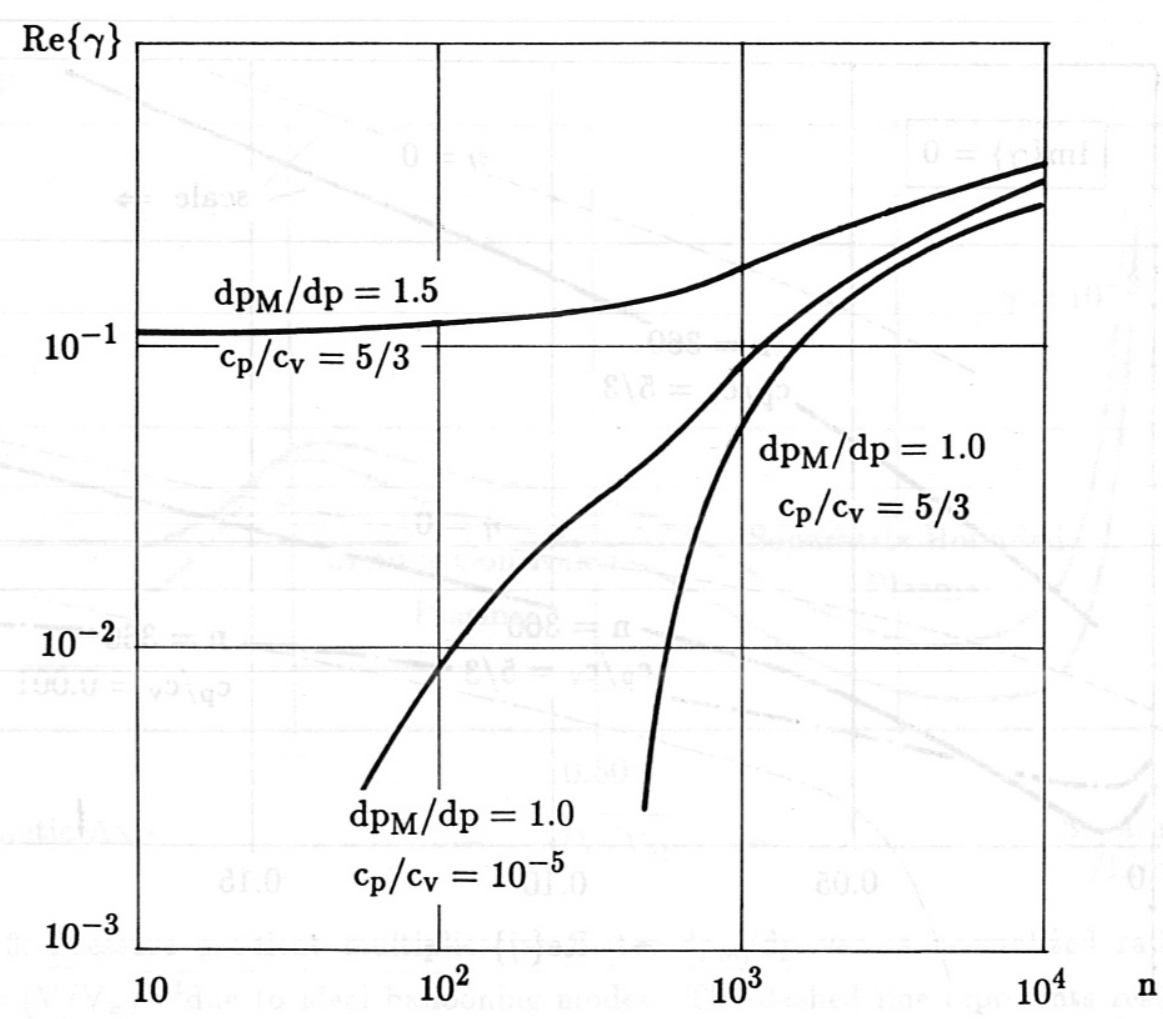

Fig. 5: Scaling of $\operatorname{Re}\{\gamma\}$ with toroidal mode number $\mathrm{n}$ for $\operatorname{Im}\{\gamma\}=0$. Results for two different pressure multiplication factors $d p_{M} / d p=1$ (lower curve) and $\mathrm{dp}_{\mathrm{M}} / \mathrm{dp}=1.5$ (upper curve) are shown. The solid line in the middle represents a calculation with vanishing compressibility and $d p_{M} / d p=1$.

From the experimental point of view interesting is the radial dependence of the growth rate for different values of $\mathrm{n}$. This is shown in Fig.6. As in the case of ideal ballooning modes (Fig.3), the most unfavourable flux surfaces with respect to stability are those with $\mathrm{r} / \mathrm{a}$-values of about 0.88. Fig.6 illustrates that in the considered range of growth rates $\operatorname{Re}\{\gamma\} \in[0.005,0.1]$ and resistivity values only modes with very large $n(n>150)$ show significant effects. For these $n$-values we don't expect the MHD treatment to be valid, since finite Larmor radius effects should be non-negligible. With an ion Larmor radius of about $0.01 \mathrm{~m}$ at ASDEX, acceptable values for the toroidal mode number are $\mathrm{n}<100$.

So far our results are for purely real growth rates. It is now interesting to investigate overstable cases and especially the question whether unstable solutions can be found for realistic values of the toroidal mode number $n(n<100)$. It is sufficient for this analysis to consider $\operatorname{Im}\{\gamma\}>0$ only, since all results are invariant under 


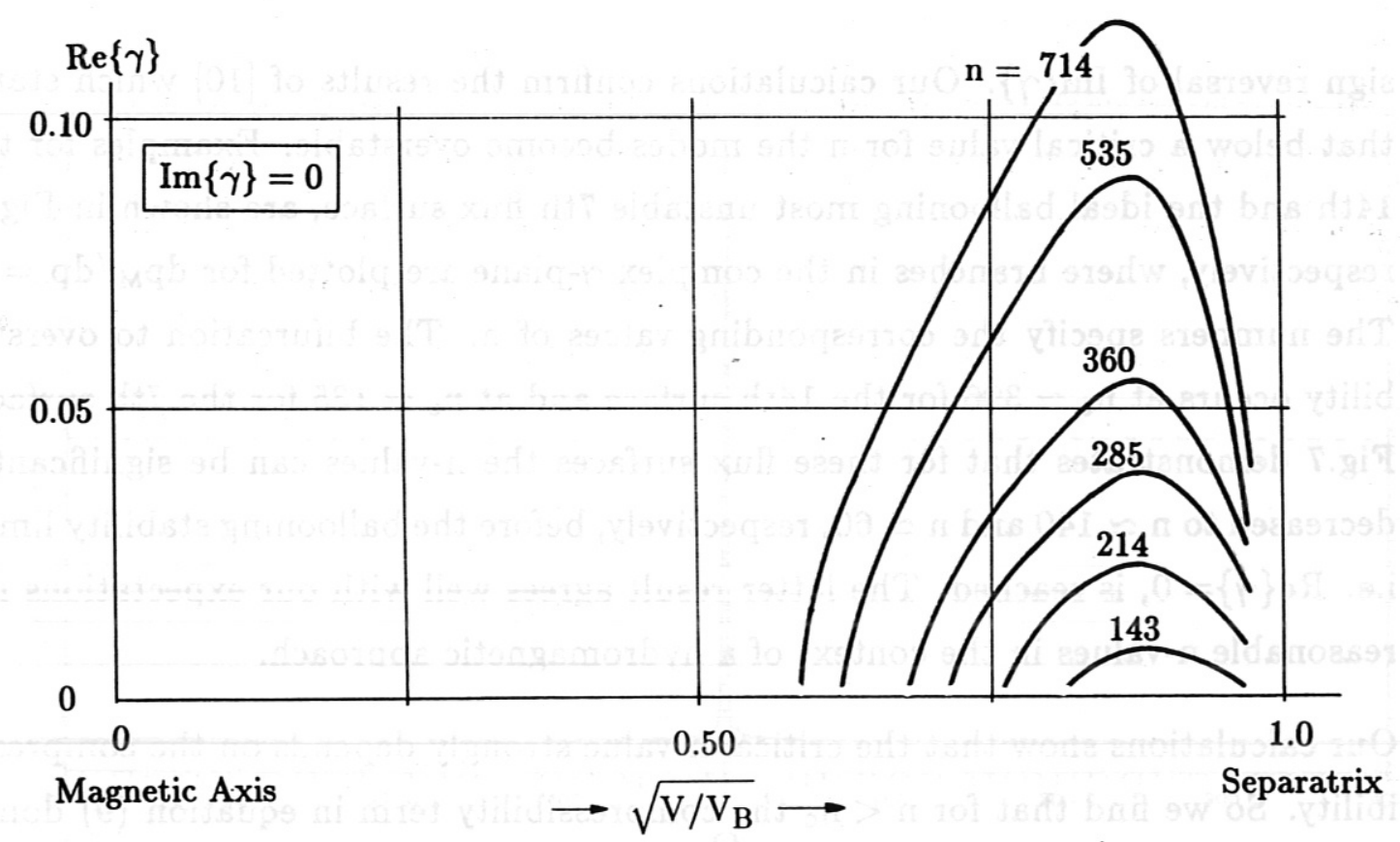

Fig. 6: Real part of the normalized growth rate versus $r / a=\left(V / V_{B}\right)^{1 / 2}$ for various toroidal mode numbers $n$ and $d p_{M} / d p=1$.

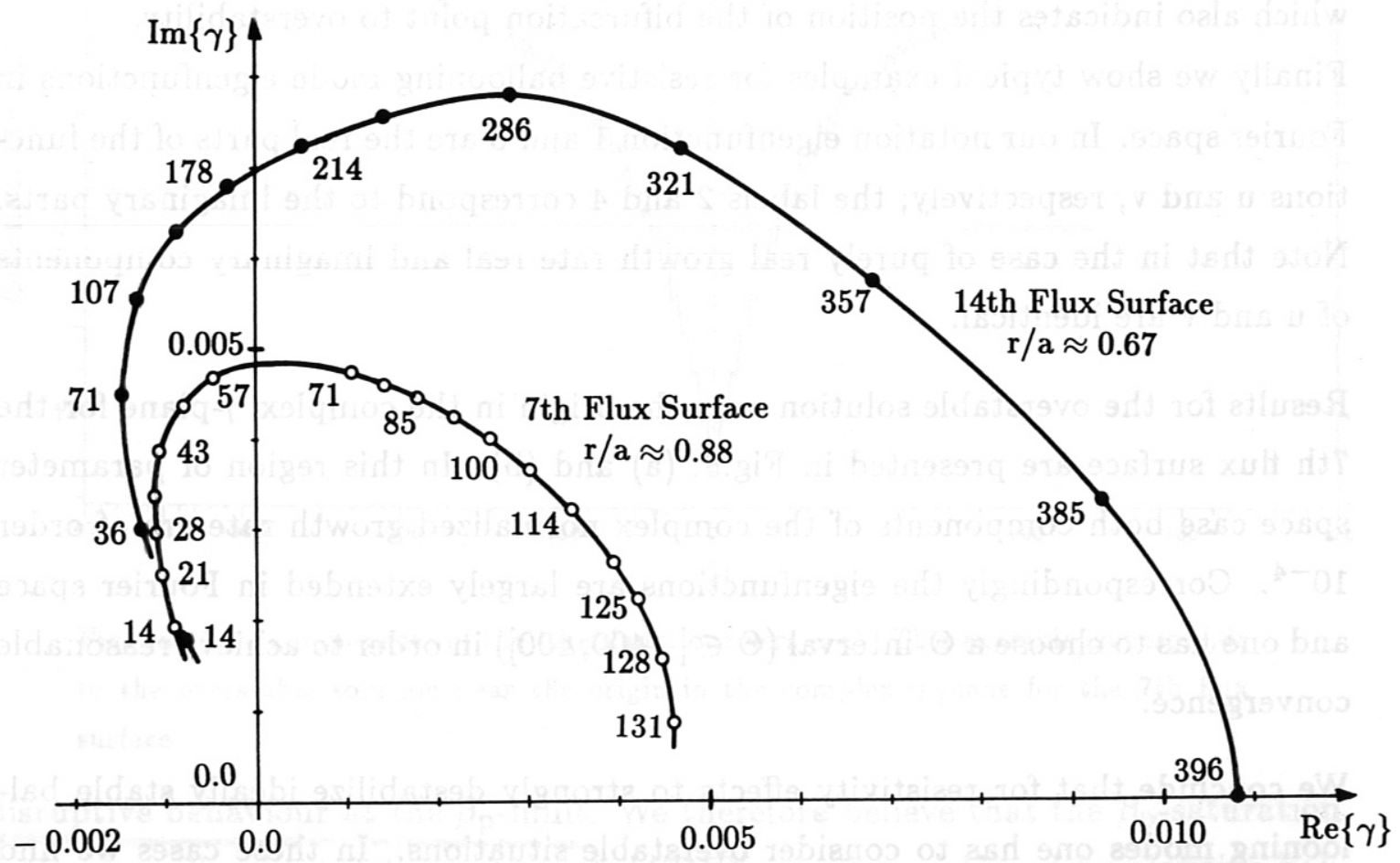

Fig. 7: Branches in the complex $\gamma$ plane for the 14 th $(r / a \simeq 0.67)$ and the 7 th flux surface $(r / a \simeq 0.88)$. The latter surface corresponds to the ideally most unstable one. Numbers at the curves label the various toroidal mode numbers. 
sign reversal of $\operatorname{Im}\{\gamma\}$. Our calculations confirm the results of [10] which states that below a critical value for $n$ the modes become overstable. Examples for the 14th and the ideal ballooning most unstable 7th flux surface, are shown in Fig.7, respectively, where branches in the complex $\gamma$-plane are plotted for $d p_{M} / d p=1$. The numbers specify the corresponding values of $\mathrm{n}$. The bifurcation to overstability occurs at $n_{c} \simeq 396$ for the 14 th surface and at $n_{c} \simeq 135$ for the 7 th surface. Fig.7 demonstrates that for these flux surfaces the $n$-values can be significantly decreased to $\mathrm{n} \simeq 140$ and $\mathrm{n} \simeq 60$, respectively, before the ballooning stability limit, i.e. $\operatorname{Re}\{\gamma\}=0$, is reached. The latter result agrees well with our expectations for reasonable $\mathrm{n}$-values in the context of a hydromagnetic approach.

Our calculations show that the critical n-value strongly depends on the compressibility. So we find that for $n<n_{c}$ the compressibility term in equation (9) dominates, whereas for $n>n_{c}$ the resistivity terms take over. Accordingly we find that with decreasing $c_{p} / c_{v}$ the critical $n$ decreases. Actually this behaviour corresponds to the dependence of the minimum in $d_{p_{M}} / d p$ versus $\operatorname{Re}\{\gamma\}$ (see Fig.4) on $c_{p} / c_{v}$, which also indicates the position of the bifurcation point to overstability.

Finally we show typical examples for resistive ballooning mode eigenfunctions in Fourier space. In our notation eigenfunction 1 and 3 are the real parts of the functions $u$ and $v$, respectively; the labels 2 and 4 correspond to the imaginary parts. Note that in the case of purely real growth rate real and imaginary components of $u$ and $v$ are identical.

Results for the overstable solution near the origin in the complex $\gamma$-plane for the 7 th flux surface are presented in Fig.8, (a) and (b). In this region of parameter space case both components of the complex normalized growth rate are of order $10^{-4}$. Correspondingly the eigenfunctions are largely extended in Fourier space and one has to choose a $\Theta$-interval $(\Theta \in[-400,400])$ in order to achieve reasonable convergence.

We conclude that for resistivity effects to strongly destabilize ideally stable ballooning modes one has to consider overstable situations. In these cases we find ASDEX discharges to be resistive ballooning unstable even at realistic values of the toroidal mode number $\mathrm{n}$. On the other hand corresponding growth rates are quite small $\operatorname{Re}\{\gamma\}<10^{-3}$ - in agreement with the experimentally observed non- 

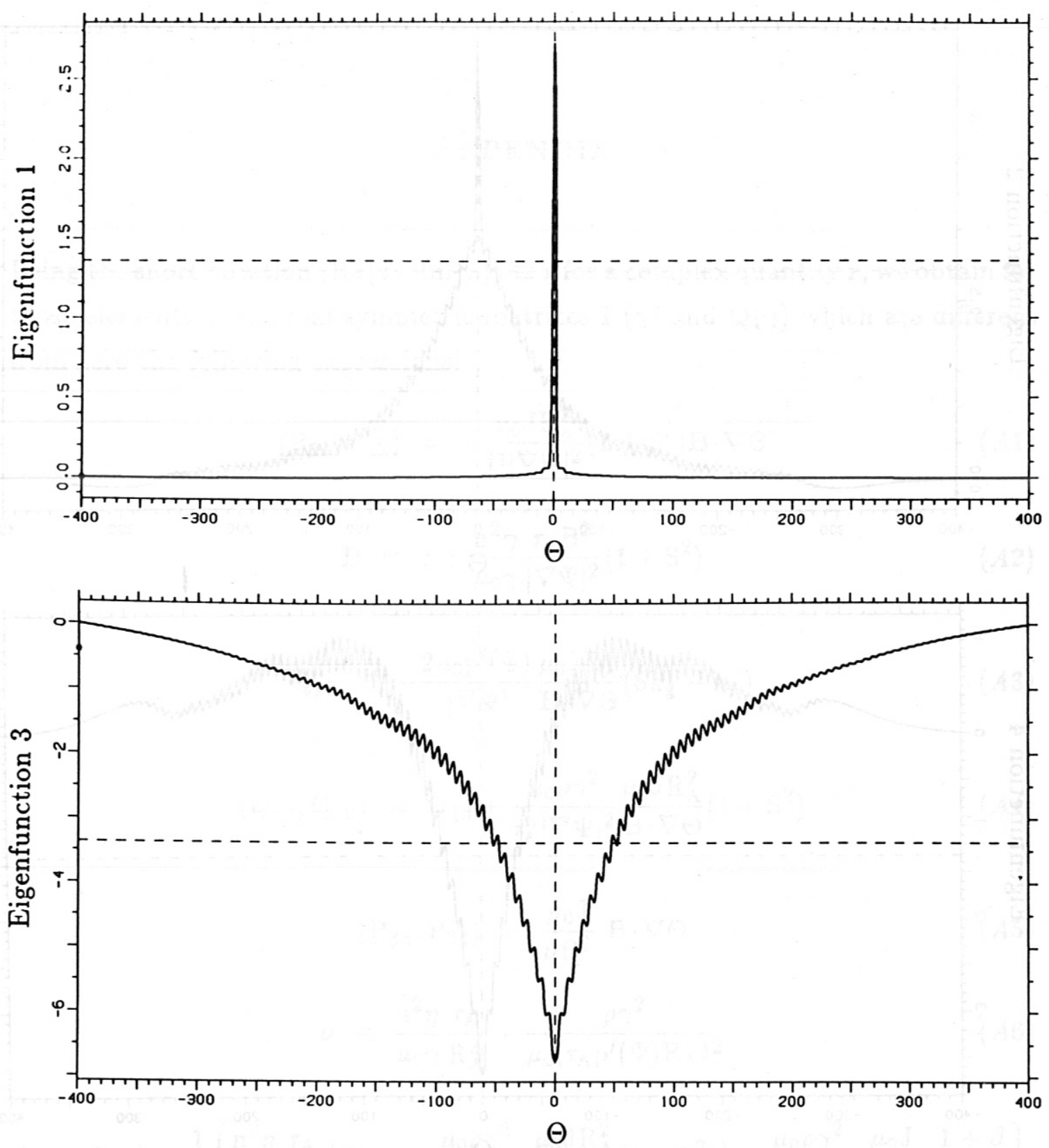

Fig. 8a: Real components of the eigenfunctions versus $\Theta$. This example corresponds to the overstable solution near the origin in the complex $\gamma$-plane for the 7 th flux surface.

disruptive behaviour at the $\beta_{\mathrm{p}}$-limit. We therefore believe that the $\beta_{\mathrm{p}}$-saturation without confinement deterioration at ASDEX, can be explained by resistive ballooning modes. 


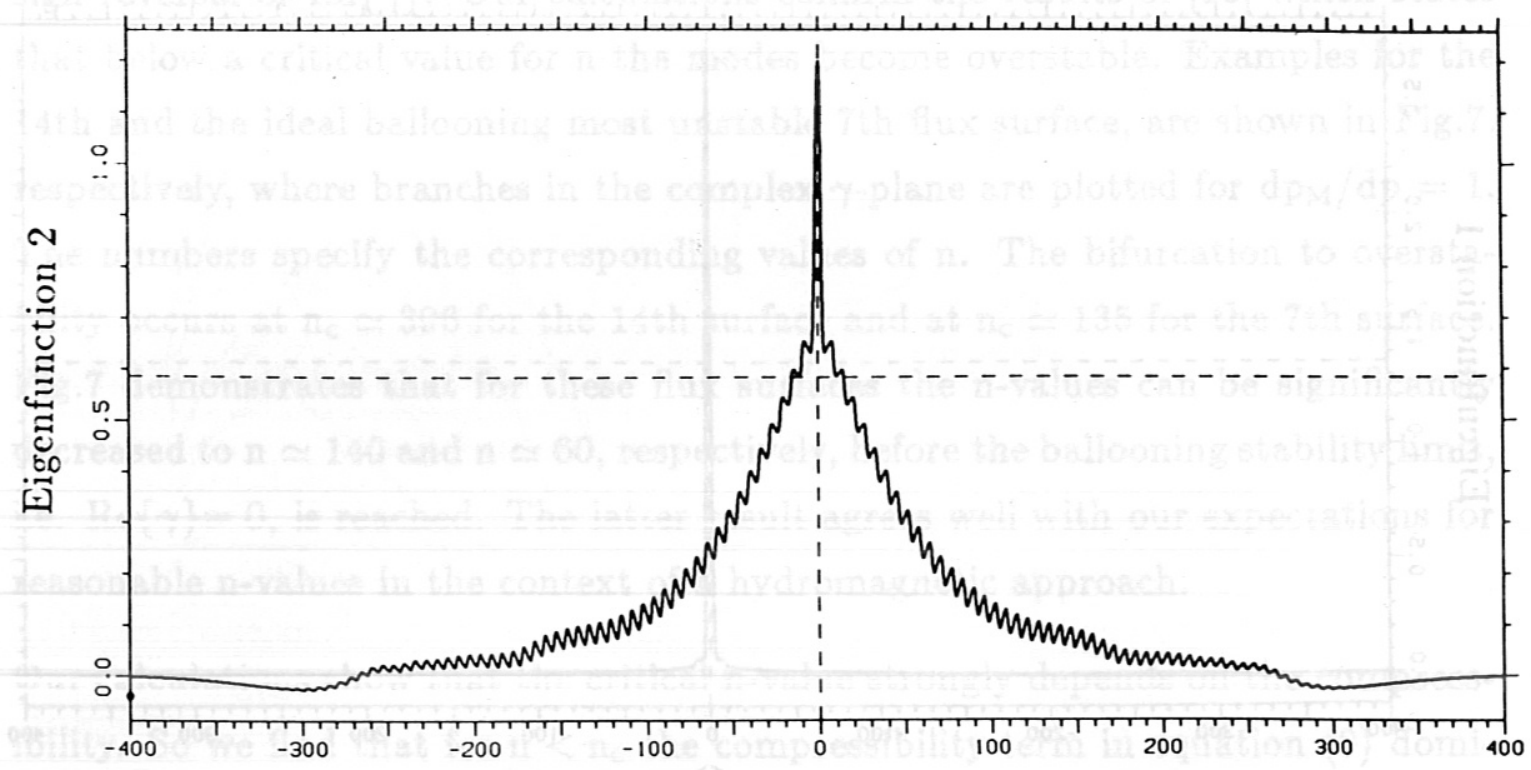

$\Theta$

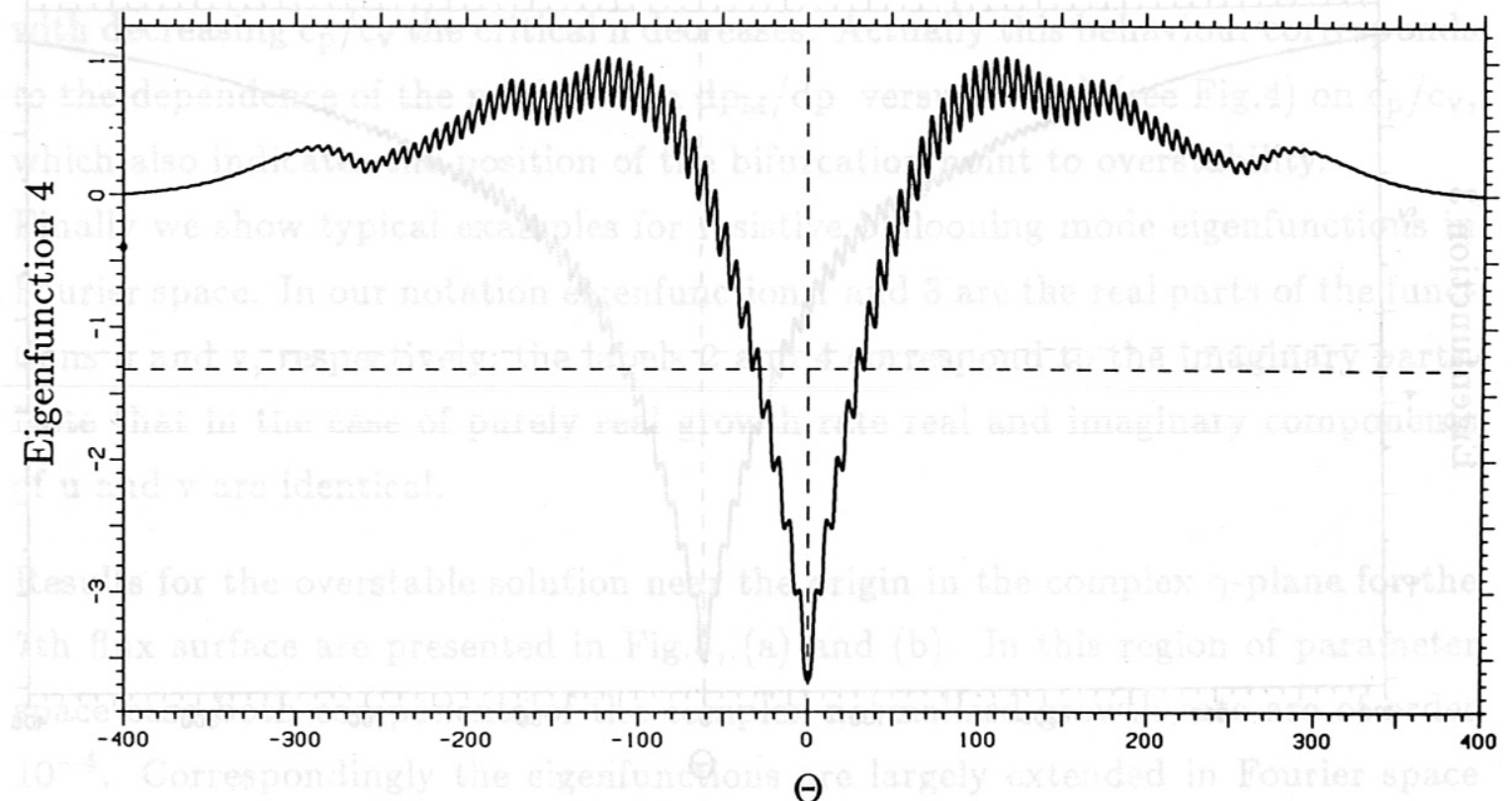

Fig. 8b: Same as Fig.8a for the imaginary components.

\section{Acknowledgements}

We would like to acknowledge useful discussions with D. Correa-Restrepo and K. Riedel. We also would like to thank $O$. Gruber for providing us with experimental results of ASDEX in TRANSP-code interpretation. 


\section{APPENDIX}

Using the short notation $(\operatorname{Re}\{z\}, \operatorname{Im}\{z\}) \equiv \mathrm{z}$ for a complex quantity z, we obtain for those elements of the real symmetric matrices $\mathbf{P}(\gamma)$ and $\mathbf{Q}(\gamma)$ which are different from zero the following expressions:

$$
\begin{aligned}
& \left(\mathrm{P}_{11}, \mathrm{P}_{12}\right)=-\frac{\mu_{0} \mathrm{JR}_{\mathrm{A}}^{2}}{\mathrm{D}|\nabla \Psi|^{2}}\left(1+\mathrm{S}^{2}\right) \mathbf{B} \cdot \nabla \Theta \\
& \mathrm{D} \equiv 1+\frac{\mathrm{n}^{2} \eta}{\mu_{0} \gamma} \frac{\tau_{\mathrm{A}} \mathrm{B}^{2}}{|\nabla \Psi|^{2}}\left(1+\mathrm{S}^{2}\right) \\
& \mathrm{Q}_{13}=-\frac{2 \mu_{0} \mathrm{p}^{\prime}(\Psi)}{|\nabla \Psi|} \frac{\mu_{0} \mathrm{JR}_{\mathrm{A}}^{2}}{\mathrm{~B} \cdot \nabla \Theta}\left(\mathrm{Sk}_{\mathrm{g}}-\mathrm{k}_{\mathrm{n}}\right) \\
& \left(\mathrm{Q}_{11}, \mathrm{Q}_{12}\right)=\mathrm{Q}_{13}+\frac{\mu_{0} \rho \gamma^{2}}{\tau_{\mathrm{A}}^{2}|\nabla \Psi|^{2}} \frac{\mu_{0} \mathrm{JR}_{\mathrm{A}}^{2}}{\mathrm{~B} \cdot \nabla \Theta}\left(1+\mathrm{S}^{2}\right) \\
& \left(\mathrm{P}_{33}, \mathrm{P}_{34}\right)=\frac{\mu_{0} \mathrm{~J}}{\nu \mathrm{B}^{2}} \mathbf{B} \cdot \nabla \Theta \\
& \nu \equiv \frac{\mathrm{n}^{2} \eta}{\mu_{0} \gamma} \frac{\tau_{\mathrm{A}}}{\mathrm{R}_{\mathrm{A}}^{2}}+\frac{\rho \gamma^{2}}{\mu_{0}\left(\tau_{\mathrm{A}} \mathrm{p}^{\prime}(\Psi) \mathrm{R}_{\mathrm{A}}\right)^{2}} \\
& \left(\mathrm{Q}_{33}, \mathrm{Q}_{34}\right)=\frac{1}{\nu}\left\{\frac{\mathrm{n}^{2} \eta}{\mu_{0} \gamma} \frac{\tau_{\mathrm{A}}}{\mathrm{R}_{\mathrm{A}}^{2}}\left(\mathrm{Q}_{13}-\frac{\mu_{0} \rho \gamma^{2}}{\tau_{\mathrm{A}}^{2}|\nabla \Psi|^{2}} \frac{\mu_{0} \mathrm{JR}_{\mathrm{A}}^{2}}{\mathrm{~B} \cdot \nabla \Theta}\left(1+\mathrm{S}^{2}\right)\right)-\frac{\mu_{0} \rho \gamma^{2}}{\tau_{\mathrm{A}}^{2} \mathrm{~B}^{2}} \frac{\mu_{0} \mathrm{~J}}{\mathrm{~B} \cdot \nabla \Theta} \frac{1+\beta}{\beta}\right\}
\end{aligned}
$$




\section{REFERENCES}

[1] O. Gruber et al., "MHD Stability and Transport of Beam Heated ASDEX Discharges in the Vicinity of the Beta Limit", 11th International Conference on Plasma Physics and Controlled Nuclear Fusion Research, Kyoto 1986, paper IAEA-CN-47/A-VI-2.

[2] F. Troyon et al., "MHD-Limits to Plasma Confinement", Plasma Phys. and Contr. Fusion 26 (1984) 209.

[3] D. Correa-Restrepo, "Resistive Ballooning Modes in Three-Dimensional Configurations", Z.Naturforsch. 37a, 848-858 (1982).

[4] S.P. Hirshman, R.J. Hawryluk, B. Birge, "Neoclassical Conductivity of a Tokamak Plasma",Nucl. Fusion 17 (1977), 611.

[5] O. Axelsson, V.A. Barker, "Finite Element Solution of Boundary Value Problems", Theory and Computation, Academic Press 1984.

[6] A Jennings, "A Compact Storage Scheme for the Solution of Symmetric Simultaneous Equations", Comput.J.9 (1966) 281-285.

[7] J.R. Bunch, L. Kaufman, "Some Stable Methods for Calculating Inertia and Solving Symmetric Linear Systems", Math.Comput. 31 (1977), 163177.

[8] H.R. Schwarz, "Methode der finiten Elemente", 2nd revised edition, B.G. Teubner Stuttgart 1984.

[9] A. George, J.W. Liu, "Computer Solution of Large Sparse Positive Definite Systems", Prentice-Hall Series in Computational Mathematics, 1981.

[10] T.C. Hender et. al., "The Effects of Compressibility of the Resistive Ballooning Mode”, Phys.Fluids 27, 1984, 1439. 